\title{
CULTURA Y DISPOSITIVOS ORGANIZACIONALES EN UNA EMPRESA FAMILIAR. ¿SATISFACCIÓN LABORAL Y/O DESGASTE PERSONAL?
}

\author{
CULTURE AND ORGANIZATIONAL DEVICES IN A FAMILY BUSINESS. JOB \\ SATISFACTION AND / OR PERSONAL BURNOUT?
}

\footnotetext{
Antonio Becerra-Hernández ${ }^{1} *$ (D); Fabiola De-Jesús Mapén-Franco ${ }^{2}$ iD; Heberto Romeo Priego-Alvarez ${ }^{1}$ (D); Aline Aleida-del-Carmen Campos-Gómez ${ }^{1}$ (iD; Diego Eduardo Menéndez-Fierros ${ }^{1}$ (iD.

1. Universidad Juárez Autónoma de Tabasco. División Académica de Ciencias de la Salud, México.

2. Universidad Juárez Autónoma de Tabasco. División Académica de Ciencias Económico-Administrativas, México.
}

*Corresponding author: Antonio Becerra-Hernández. e-mail: becerra_antonio@hotmail.com

\section{RESUMEN}

Objetivo: el estudio examinó el entrecruzamiento de determinismos sociales y psíquicos del empleado con el entramado ideológico de gestión de una empresa familiar desde la sociología clínica del trabajo. Material y método: se acudió a un enfoque cualitativointerpretativo, con entrevistas, revisión del manual organizacional y análisis clínico del trabajo. Resultados: los hallazgos indican que los integrantes, familiares consanguíneos o no, asumen responsabilidades y replican conductas y comportamientos al interior de la compañía, para cumplir con las metas y compromisos que la empresa les transmite a través de sus dispositivos de poder. Discusión: la aplicación de instrumentos organizacionales implementados desde la ideología gerencial, como, la misión, visión y valores, permite satisfacer ilusiones de reconocimiento, omnipotencia y oportunidades. Conclusiones: es posible analizar la interrelación sociopsicológica del sujeto insertado en una organización empresarial, invistiéndola libidinalmente y vinculándose afectivamente con ésta.

Palabras clave: Dispositivos organizacionales; sociología clínica del trabajo; teoría organizacional; cultura organizacional; empresa familiar.

Cómo citar:

Becerra-Hernández, Antonio; Mapén-Franco, Fabiola De-Jesús; Priego-Alvarez, Heberto Romeo; Campos-Gómez, Aline Aleida-del-Carmen; Menéndez-Fierros, Diego Eduardo. (2021). CULTURA Y DISPOSITIVOS ORGANIZACIONALES EN UNA EMPRESA FAMILIAR. ¿SATISFACCIÓN LABORAL Y/O DESGASTE PERSONAL?. Revista de Investigaciones Universidad del Quindio, 33(1), 231-240. https://doi.org/10.33975/riuq.vol33n1.583 


\section{ABSTRACT}

Objective: the study examined the interweaving of social and psychic determinism of the employee with the ideological framework of the management of a family business from the clinical sociology of work. Material and method: a qualitative-interpretive approach was used, with interviews, review of the organizational manual and clinical analysis of the work. Results: the findings indicate that the members, blood relatives or not, assume responsibilities and replicate behaviors and behaviors within the company, to meet the goals and commitments that the company transmits to them through its power devices. Discussion: the application of organizational instruments implemented from the managerial ideology, such as mission, vision and values, allows satisfying illusions of recognition, omnipotence and opportunities. Conclusions: it is possible to analyze the socio-psychological interrelation of the subject inserted in a business organization, investing it libidinally and bonding with it.

Keywords: Organizational devices; clinical sociology of work; organizational theory; organizational culture; family business.

\section{INTRODUCCIÓN}

La punzante queja respecto del sufrimiento, la multiplicación de los síntomas depresivos, el sentimiento generalizado de hostigamiento, la hiperactividad, el agotamiento profesional y el desarrollo de suicidios en el lugar de trabajo constituyen otros síntomas de un malestar profundo. "No se volvían locos todos, pero todos estaban afectados".

Vincent de Gaulejac, Existir en un mundo paradójico

La administración es tan antigua como el mismo hombre. Como ciencia, "esta disciplina tiene sus orígenes a finales del siglo XVIII, con el inicio de la Revolución Industrial" (Amaru, 2010). Lo que en un principio se fabricaba de manera artesanal y con la baja probabilidad de que dos artículos fueran similares, las líneas de producción o bandas de montaje lo hicieron posible, gracias a la implementación de estrategias administrativas y de operación donde la ingeniería, el diseño industrial, la administración, entre otras, jugaron un papel fundamental para ello. Taylor y Ford, fueron algunos de los que aportaron y concretaron ideas específicas para el diseño de nuevas formas de trabajo en la etapa moderna de la industria.
Con Ford al frente de la industria automotriz norteamericana (Friedmann, 1977), se establece el nuevo artesano fordiano, "aquel obrero que se encontraba en la línea de montaje realizando tareas rutinarias con tiempos y movimientos perfectamente medidos y controlados". Sin embargo, a partir de 1914 (Quiroz, 2010) se presenta la batalla contra los excesos del taylorismo $\mathrm{y}$ el fordismo. El ciclo de protestas inicia en Estados Unidos con el ausentismo laboral y la rotación de personal. Más tarde, esporádicamente, al sabotaje y al ausentismo se unirían los paros y las huelgas, taylorismo y fordismo estaban siendo cuestionados. Años después, Elton Mayo (1972), enfocó sus modificaciones al proceso laboral, centrándose en los trabajadores y sus necesidades subjetivas, convirtiéndose en el principal precursor de las llamadas relaciones humanas, para disminuir las presiones de trabajo y las jornadas extenuantes de empresas industrializadas, que, gracias a la estandarización de los procesos, hicieron posible que los trabajadores fueran reemplazables $y$, en consecuencia, explotables. El Movimiento de Relaciones Humanas de la década de los 30's, popularizó la creencia de que los trabajadores felices eran en promedio más productivos que los trabajadores menos felices o infelices ( Cropanzano y Wright, 2001; Wright, Cropanzano y Bonnett, 2007, citados por Salgado, Blanco y Moscoso, 2019). 
Sin embargo, la buena intención poco duro en el espacio de las organizaciones empresariales. La era moderna cargó consigo la automatización de los procesos. Las telecomunicaciones y el desarrollo de sistemas computacionales agilizaron las comunicaciones y el procesamiento de información a alta escala; la robótica, junto con los llamados grupos de mejora continua o círculos de calidad, ayudaron a optimizar los tiempos de fabricación y a reducir el número de productos defectuosos. Empero, en el fondo, la exigencia hacia el empleado se incrementó; se le impuso lograr metas cuyo propósito de alcanzar ponían en riesgo su propia salud física y emocional. La sobredosis laboral o síndrome conocido como "karoshi" -muerte o inhabilidad permanente por enfermedades cerebro-vasculares y enfermedades isquémicas del corazón como resultado del exceso de trabajo-, en países como Japón, India, Corea del Sur, Taiwán, China, Europa, Estados Unidos de Norteamérica, son reconocidos ya como un problema de salud. La cifra de muertes o padecimientos cuyas causas se relacionan con la exigencia en las áreas laborales -hipertensión, depresión, infartos cardiacos y cerebrales, suicidio o intentos suicidas, entre otros-, llevan a considerar que la etapa posmoderna de la exigencia en búsqueda de la mejora continua, la calidad, la satisfacción del cliente, el cero rechazos de productos o de quejas del cliente, se entrecruzan con aquellas necesidades que el propio sujeto hace suyas a partir de los determinismos sociales y psíquicos que le dan significado a su condición de "ser humano"; éstas son: la oferta de satisfacciones ilimitadas de reconocimiento, de omnipotencia y de oportunidades, la auto confirmación de ser capaz y de poder alcanzar las metas impuestas, la necesidad del poder y el dinero para satisfacer el consumismo impuesto por las ideologías predominantes. Todo ello, probablemente, sin estar consciente de que su salud física y emocional se encuentran en riesgo. Como lo señala De Gaulejac (2010), "la ideología gerencial considera al individuo como un recurso más que como un sujeto. Entonces, el individuo es instrumentalizado al servicio de objetivos financieros, operatorios, técnicos, que le hacen perder el sentido de su acción, incluso el sentido de su existencia". A diferencia del taylorismo, donde el foco es el control del cuerpo, en el escenario contemporáneo el foco de la gestión es la subjetividad del individuo. Los individuos deben alinear sus aspiraciones con los objetivos económicos. Esta ideología gerencial puede asegurarse a través de lo que se ha denominado cultura organizacional.

\section{MARCO TEÓRICO}

\section{Cultura organizacional}

Para el concepto de cultura organizacional (CO) existen diversas aproximaciones. Según Reyes y Moros (2019), "laCOesparteconstitutiva, esencial y de la propia naturaleza de la organización, no es estática, es de naturaleza dinámica y fluctúa en función de múltiples factores, siendo los más representativos aquellos relacionados con la dirección, las diferentes subculturas que emergen, las condiciones macroeconómicas y sociales en las que se inserta la organización y los elementos discursivos que contribuyen a generar un sentido de identidad determinado". Anzola (2003), lo define como "un conjunto de prácticas sociales materiales e inmateriales que dan cuenta de las características que distinguen a una comunidad ya que establecen una atmósfera afectiva común y un marco cognitivo compartido". Para Schein (2004), la CO es "un patrón de supuestos básicos compartidos que el grupo aprende conforme resuelve problemas de adaptación externa e integración interna, que funcionan suficientemente bien para ser considerados válidos $\mathrm{y}$, por tanto, ser enseñados a nuevos miembros como la forma correcta de percibir, pensar y sentir con relación a esos problemas". Según Garibaldi, Wetzel y Ferreira (2009), la CO es un "conjunto de significados y valores que los miembros de una organización tienen. Estos significados y valores son los bloques de construcción de cultura organizacional y se expresan mediante símbolos, comportamientos y estructura". Cheung, Wong y Lam (2012, citados por Rivera, Carrillo, Forgiony, et al., 2018), se refieren a la 
CO como "el patrón de supuestos básicos que se manifiestan a través del comportamiento de una organización". Para Sampieri, Valencia y Soto (2014), se trata de "un recurso estratégico que posee el valor de asegurar la continuidad y permanencia de las organizaciones". En cuanto a sus beneficios, Palafox, Ochoa y Jacobo (2019) señalan que la cultura "es un aspecto fundamental de las organizaciones, pues gestionándola correctamente y de la mejor manera posible, permite conseguir un buen ambiente y clima laboral, además de que los trabajadores se sientan orgullosos de laborar en las organizaciones". Sin embargo, como lo señalan Chernyak-Hai y Tziner (2016), "un clima organizacional percibido desfavorablemente se relaciona claramente con informes más altos de conflictos entre el trabajo y la familia. Esta influencia puede deberse a la percepción general de que el entorno organizacional de uno es menos solidario de lo preferido y, por lo tanto, el conflicto, a saber, el desequilibrio entre las demandas laborales y familiares, se amplifica".

En ese mismo sentido, Esquivel, Martínez y Silva (2020), señalan que se debe concebir que un clima [organizacional] positivo favorece el cumplimiento de los objetivos generales que percibe la empresa... Por el contrario, un clima negativo supone una falta de identificación con los objetivos y destruye el ambiente de trabajo, lo que ocasiona situaciones de conflicto y de bajo rendimiento. Es la alta dirección, con su cultura y sistemas de gestión, la que propicia o no el terreno adecuado para un buen clima y forma parte de las políticas de recursos humanos, la mejora de ese ambiente, con el uso de técnicas precisas".

La construcción y reproducción de la cultura organizacional tiene cabida en cualquier organización empresarial.

\section{Empresa familiar}

Al igual que el concepto de cultura organizacional, el de empresa familiar (EF) es variable y depende de distintas condiciones o características para referirse a él. Sin embargo, la EF tiene al menos tres componentes que la conforman: la propiedad, la empresa y la familia. Según Donnelley (1964):

"Una EF puede considerase como tal solo si la familia está vinculada a la empresa por el menos por dos generaciones y si estas conexiones familiares ejercen influencia acerca de los intereses y objetivos de la empresa... Los valores y las creencias de la empresa tienen que identificarse con los de la familia, las acciones de un familiar impactan en la imagen y reputación de la empresa y el puesto que ocupa el miembro de la familia influye en su situación familiar".

Gallo y Sveen (1991), exponen que una EF es aquella en la que una sola familia posee la mayoría del capital y tiene un control total. Los miembros de la familia también forman parte de la dirección y toman las decisiones más importantes".

Por otra parte, de acuerdo con De la Garza (et al., 2011), "los valores en las EF son un factor clave en el desempeño de dichas organizaciones y logran convertirse en una ventaja competitiva que se puede conservar a lo largo del tiempo". Según Molina, Botero y Montoya (2016), "los que mejor describen tanto a la familia como a la generación que controla la empresa son el compromiso, la responsabilidad, la justicia o equidad, la credibilidad, el respeto a la legalidad y el trabajo duro".

\section{La teoría organizacional -el empleado- y la sociología clínica del trabajo -el ser del hombre y el ser de la sociedad.}

Para la teoría organizacional los procesos intersubjetivos que se presentan en las organizaciones están descartados desde la óptica racional-instrumental $\mathrm{y}$, por lo tanto, el componente socio-afectivo del individuoempleado o colaborador- queda desligado de la gestión tangible y cuantificable del negocio. Consecuentemente, elementos, tales como, la misión, la visión o el enunciado de valores institucionales, forman parte de los dispositivos 
estratégicos utilizados por las organizaciones empresariales, en la intención de alcanzar las metas y objetivos empresariales. Sin embargo, en contraste, Guerrero y De Gaulejac (2017) señalan que "el sujeto en la organización, equipado con todas sus fantasías, miedos, ilusiones, proyecciones, identificaciones y mecanismos de poder podrá alinearse a los objetivos económicos de la organización, quien a su vez dará las condiciones para que este pueda extender sus límites y satisfacer sus aspiraciones de reconocimiento y de omnipotencia, proyectando en la empresa todas las cualidades que quisiera tener como propias".

Por tanto, suponer una separación de lo que racionalmente indica una organización formalmente constituida de aquello emocional en el ser humano, implicaría solo una posición cosificante cuya intención puede colocarse en duda al analizar las conductas y comportamientos de los integrantes de cualquier organización humana.

\section{Propósitos y contribuciones de este estudio}

Considerando lo antes señalado, el objetivo del estudio es examinar el entrecruzamiento de determinismos sociales y psíquicos del empleado con el entramado ideológico de gestión de una empresa familiar. Su aportación se relaciona con la identificación de estrategias y tácticas de la empresa familiar, diseñadas a partir de la ideología de gestión -cultura organizacional, identidad e imagen-, para asegurar la permanencia y continuidad organizacional, engarzadas con la necesidad latente o manifiesta del empleado, con el fin de aspirar a satisfacer sus aspiraciones de reconocimiento y de omnipotencia, proyectando en la empresa todas las cualidades que quisiera tener como propias, ya que como lo señala De Gaulejac (2005), "la particularidad de la gestión [empresarial] reside en el hecho de que no se trata de perseguir una finalidad elegida por los individuos, ni una finalidad negociada al interior de una colectividad, sino una finalidad impuesta desde el exterior".

\section{METODOLOGÍA}

\section{Participantes}

La organización empresarial de laboratorio de análisis clínicos se fundó en 1963 (Becerra, Menéndez y Mapén, 2019), en el sureste de México. Los 70 empleados formalmente contratados se encontraban distribuidos entre la casa matriz y las sucursales. Los integrantes que formalmente representaban a la familia empresaria eran cinco: el dueño fundador, sus dos hijos y sus respectivas esposas, todos ellos con estudios de químicos farmacobiólogos.

En lo que se refiere a la organización formal, se trató de una estructura simple, ya que están divididas en tres direcciones: la dirección general (dueño fundador), la administrativa (hijo mayor propietario) y la operativa (hijo menor propietario). Dentro de los servicios que ofrecía la empresa, se encontraban los siguientes: análisis de rutina, pruebas especiales, células madre, tamiz neonatal, andrología y fertilización, pruebas de paternidad, atención a empresas y maquila a otros laboratorios

\section{Procedimiento}

A continuación, se muestran los enunciados de misión y visión empresarial (Becerra, Menéndez y Mapén, 2019), y valores, que forman parte de su estrategia de identidad corporativa, además de algunos fragmentos de las entrevistas con los propietarios o empleados. Estos dispositivos son analizados y contrastados desde la lógica racional-instrumental de la teoría organizacional vs. la perspectiva de la sociología clínica del trabajo.

\section{RESULTADOS}

\section{Misión}

"Ser una empresa que ofrece al público en general la realización de procesos del laboratorio de análisis clínicos, cuyos resultados permiten 
satisfacer sus necesidades en el ámbito de la calidad, seguridad y control de los resultados".

Para Urbiola y Levin (2018), "la organización, como un sistema socio-tecnológico, indica que un grupo de personas pueden desarrollar los objetivos, las metas, la visión y la misión organizacional para que estos sean difundidos en espacios apropiados, empleando estrategias y mecanismos para dar a conocer, aprender y reproducir la cultura corporativa". Estos espacios pueden ser todos aquellos que estén al alcance de la propia organización, tales como, los cursos de inducción, las juntas de trabajo, los correos electrónicos, los folletos institucionales, entre otros. El aprendizaje y reforzamiento de la cultura organizacional asegura la reproducción de lo que la empresa desea observar en sus empleados. La intención encaminada hacia la calidad, seguridad y control de los resultados, pueden indicarnos que permanecen en sus actividades la búsqueda de logros personales y profesionales que están sirviendo como impulsores de sus metas y objetivos empresariales.

\section{Visión}

"Mantener al laboratorio de análisis clínicos como líder en el sur-sureste de la república mexicana, ofreciendo calidad en todos sus servicios, contribuyendo en la prevención y diagnóstico preciso y oportuno de los pacientes".

Las empresas actualesacudendeformapermanente a la enunciación de estas reglas o valores que pretendan alinear los comportamientos de quienes en ellas permanecen o de los que arriben. De tal suerte que, la empresa indica las reglas del juego, lo que se permite y lo que se castiga; el empleado, será quién asuma el compromiso de adherirse a lo prescrito por sus superiores, quienes en este caso desempeñan la figura de autoridad.

Teniendo como fuente de consulta el manual organizacional, a continuación, se describen los valores organizacionales que la empresa objeto de estudio ha establecido como parte de su cultura organizacional. Enseguida, se incorporan fragmentos de las entrevistas con los propietarios o algunos empleados.

\section{Valores institucionales de la empresa familiar}

\section{a) Responsabilidad}

"Tienes que ser papá hasta que te mueras, mamá hasta que te mueras. En la manera en que des, vas a recibir. Mis papás me dieron un buen sustento de educación. Esas cosas funcionan si las aplicas tanto en la familia, como en la empresa" (Director general fundador).

"Al final de cuentas, somos un engrane. Procuramos trabajar para llegar siempre a la meta. La integración ayuda a llegar al objetivo" (Gerente administrativo).

Aquí el individuo asume un mandato, forma parte de una red que alinea los objetivos de sus integrantes a uno principal. Se trata de entender y atender dicho mandato y "responder" por éste, sumando los esfuerzos individuales, que pueden estar asociados a un sentimiento de culpa si aquel no es alcanzado por haber faltado a ese compromiso.

\section{b) Honestidad}

"La honestidad es otro valor igual de importante para nosotros en la empresa. Va más allá de no cobrarle demás a un paciente, sino al hecho de que, si no sabemos acerca de un estudio que solicita un médico, tenemos como política y principio decirle al paciente que van a investigar al respecto y después realizan el estudio y entregan un resultado como debe ser. No engañamos a la gente" (Hermano mayor propietario).

La honestidad alude al hecho principalmente de actuar de forma coherente y congruente; pensar, decir y hacer las cosas en apego a los principios de verdad y de justicia. Puede asociarse evidentemente con situaciones que tienen que ver con la posibilidad de otorgarle al 
cliente un servicio honesto y conducirse dentro de la empresa con rectitud y honradez para no dañar los intereses de ésta y conservar su empleo. Implica, además, un respeto por lo que se dice y se hace.

\section{c) Mejora continua}

"Si existe algún problema, busco resolverlo platicando, checamos en dónde fallamos y buscamos corregirlo. Mejorar nuestra carencia y buscar mejorar y crecer a futuro. Vamos evolucionando como área $\mathrm{y}$, como empresa, caminamos por el mismo objetivo" (Encargado de sistemas).

Fortino, Jeantet y Tcholakova (2015), indicaron que, como parte de sus actividades profesionales diarias, "el trabajo moviliza a las personas en su ‘todo', cuerpo y alma, alentándolos a confiar en habilidades técnicas, pero también emocionales, para atraer su entusiasmo y su deseo de hacerlo bien, para movilizar empatía y confianza".

Sin embargo, es posible interpretar que asumiendo la imperfección o el implacable deseo de "ser mejores cada día", esa llamada búsqueda de la mejora continua se convierte en el mecanismo persecutorio de la demanda hacia la perfección. Como práctica administrativa, sirve para resolver algunos problemas y contribuye a incrementar las ganancias de las empresas; no obstante, en el plano individual ha desatado una serie de complicaciones asociados a la salud de los empleados y a la estabilidad socio-emocional $\mathrm{y}$ física.

\section{d) Pasión por el servicio}

"Lo primero que identifican los clientes es el servicio, ya que, para ellos, una empresa que brinda un buen servicio es lo que la gente reconoce, es lo primordial. Otro punto sería la calidad" (Hermano menor propietario).

El servicio como valor se acompaña de un compromiso del empleado consigo mismo y con sus clientes, asociado al entusiasmo por atenderlos con cortesía y amabilidad, descartando cualquier posibilidad de discriminación o diferencia según la persona que recibe el servicio. La pasión por el servicio conlleva rasgos de sublimación que inducen al propietario o trabajador a dar lo mejor de sí.

\section{e) Honradez}

"Si el paciente es de un médico amigo de mi hermano, yo le hago los estudios y le paso el cobro a mi hermano" (Hermano mayor propietario).

La honradez en el ámbito laboral puede circunscribirse a la importancia de que el empleado deba manejarse con integridad y a ejecutar el trabajo indicado correctamente (con probidad), lo cual le permite ganarse la confianza de sus superiores y de sus compañeros de trabajo. Subyace en ella la exigencia para la persona de guardar celosamente lo que se le confiere y cuidar de su integridad para enaltecer su figura. De esta manera, el empleado introyecta cualidades asociadas a la transparencia de sus actos para estar en posibilidades de pertenecer a un grupo que le otorgue seguridad y aceptación, representado en este caso por la empresa.

\section{f) Compromiso}

"Yo siento que todos encajamos. Cada persona está comprometida con su trabajo". El eslogan de la empresa lo tenemos muy metido en nuestra cabeza. Hacer bien las cosas. Hay compromiso, todos trabajamos profesionalmente" (Laboratorista).

El compromiso en este contexto queda entendido como el esfuerzo para realizar el trabajo por el que se fue contratado, además de estar en condiciones de "dar el extra" cuando la situación así lo requiera, independientemente de que se haya rubricado un instrumento legal que formalice la relación empresa-empleado.

El conjunto de valores previamente abordados, pueden ser ubicados en el desempeño contextual 
(Organ, 1998, citado por Palenzuela, Delgado y Rodríguez, 2019), y éste definirse como "la expresión de un comportamiento benevolente y voluntario hacia la organización, que está más allá de las obligaciones formales de un trabajador y que la organización para la que trabaja no reconoce directamente", ya que "los empleados contribuyen a la efectividad organizacional haciendo cosas que no están definidas dentro de sus funciones principales, pero que son importantes porque dan forma a los objetivos de la organización y el contexto social que apoya las actividades" (Borman y Motowidlo, 1993, citados por Palenzuela, Delgado y Rodríguez, 2019).

\section{DISCUSIÓN}

Para De Gaulejac (2011, citado por Maugeri, 2012) "la organización se considera como un fenómeno 'sociopsíquico', es decir, una construcción social sustentada por procesos psíquicos (proyección, introyección, idealización, sublimación, etc.), cuyas fuentes son explotadas más o menos voluntariamente por la gerencia para impulsar su acción". Por lo tanto, sugerimos que los mecanismos racionales de cualquiera de las organizaciones empresariales modernas indican, entre otros aspectos, la pretensión de un alineamiento hacia lo ideal, aquello que desde la máxima autoridad de la empresa se inscribe como un posicionamiento que habrá de alcanzarse y que exige el apego a las normas para lograr sus objetivos. La satisfacción del cliente, la calidad de los servicios y el compromiso, conllevan una carga racional y emocional importante de obligación del empleado hacia la empresa, para que ésta logre los resultados económicos esperados. Bargsted, Ramírez-Vielma y Yeves, 2019, indican que "las creencias personales sobre las competencias personales (autoeficacia) podrían ser un fuerte motivador del comportamiento que tiene un impacto en los resultados del trabajo al hacer que los empleados tengan más confianza para resolver conflictos, superar las frustraciones y persistir contra dificultades y, por lo tanto, estar más satisfechos con su desempeño laboral".
Por el lado de los valores, éstos son promovidos desde un espacio racional-económico, pero también pueden ser trasladados a otras esferas sociales dónde los individuos participan.

Es evidente que los rasgos normativos forman parte del abanico de posibilidades que el ser humano tiene para asumir un rol y desenvolverse en la sociedad como ella lo requiere; así, los valores lo son en tanto se convierten en fragmentos de los mecanismos restrictores de conductas no aceptadas socialmente, o de los promotores de las aspiraciones y deseos individuales. El respeto, la honestidad, la mejora continua, la responsabilidad, entre otros, se sitúan en el andamiaje social del individuo de la empresa y sirven como códigos escritos o las reglas del juego para ser practicados y conservar su permanencia en la organización objeto de estudio. La organización empresarial espera que sus empleados muestren las conductas y comportamientos asociados a esos valores difundidos como parte de la estrategia de comunicación.

Es posible señalar que se trata de una intención racional y latente para sublimar y sustituir, por medio de actividades "productivas" -en el sentido racional instrumental de la ideología gerencial-, los deseos inconscientes de origen, para "entregarse en cuerpo y alma", y aspirar a la posibilidad de ver cubiertas sus expectativas y necesidades personales. Para Guerrero y De Gaulejac (2017), "la empresa a través de sus dispositivos organizacionales comunica una imagen de omnipotencia y excelencia que el empleado introyecta, y proyecta todas las cualidades que quisiera tener como propias. El ideal del yo del colaborador encuentra en la empresa una formidable caja de resonancia para extender sus límites y satisfacer sus aspiraciones de reconocimiento y de omnipotencia”.

La empresa familiar, como una modalidad de una organización formal, reúne los atributos de cualquier organización y protege a sus integrantes (consanguíneos o no) contra aquello que represente caos, confusión, ambivalencia 
y ansiedad, colectivamente proyectados de las mentes individuales hacia las organizaciones, apoyándose en dispositivos instrumentales, tales como, la misión, la visión y valores organizacionales, para legitimar su razón de ser. En este sentido, la organización empresarial realiza al menos una doble acción: contiene aquellas fantasías individuales de sus integrantes (propietarios y empleados) a través de elementos racionales, y agrupa recursos y medios de poder para la realización de objetivos de producción de bienes o servicios, o con fines de orden social. "La gestión es en definitiva un sistema de organización del poder. Detrás de su neutralidad aparente, debemos comprender los fundamentos y las características de este poder" (De Gaulejac, 2005).

Por último, los autores juzgan pertinente indicar que los hallazgos y el análisis del estudio de caso no permiten establecer generalizaciones, ya que en concordancia con Navarro, Roe y Artiles (2015) “éstos solo son válidos para el episodio particular durante el cual se realizan las observaciones internas de los participantes, y para el mapeo específico de ese episodio dentro de la vida de los participantes". Sin embargo, sí abren la posibilidad para que se efectúe a partir de disciplinas emergentes como la sociología clínica del trabajo, el análisis de las articulaciones entre los determinismos sociales y los psíquicos; se estudie el campo de la exclusión social o se realicen trabajos con los grupos de implicación e investigación. Lo anterior, podrá contribuir con posibles explicaciones a fenómenos sociales gestados en el interior de las organizaciones humanas y sugerir probablemente la implementación de algunas estrategias o acciones preventivas.

\section{REFERENCIAS}

1. Amaru, A.C (2010) Fundamentos de la Administración. Teoría General y Proceso Administrativo. Pearson Educación México, S. A. de C.V.

2. Anzola, O. (2003). Una mirada a la cultura corporativa. Bogotá: Universidad Externado de Colombia.

3. Bargsted, M., Ramírez-Vielma, R., y Yeves, J. (2019). Autoeficacia profesional y satisfacción laboral: el papel mediador del diseño del trabajo. Revista de Psicología del Trabajo y de las Organizaciones, 35, 157-163. https://doi.org/10.5093/jwop2019a18

4. Becerra, A., Menéndez, D. E. y Mapén, F. (2019). La Familia, Grupo Social Permanente en las Organizaciones desde una Perspectiva Psicoanalítica [The Family, Permanent Social Group in Organizations from a Psychoanalytic Perspective]. Acción Psicológica, 16(2), 91-102. https://doi. org/10.5944/ap.16.2.25383

5. Chernyak-Hai, L. y Tziner, A. (2016). The "I believe" and the "I invest" of work-family balance: The indirect influences of personal values and work engagement via perceived organizational climate and workplace burnout. Revista de Psicología del Trabajo y de las Organizaciones, 32(1), 1-10. https://dx.doi.org/10.1016/j.rpto.2015.11.004

6. De Gaulejac, V. (2010) Existir en un mundo paradójico. Vol. 10 Núm. 20: 21-42. Administración y Organizaciones. Recuperado de: https://rayo.xoc.uam.mx/index.php/Rayo/article/view/215

7. De Gaulejac, V. (2005). La société malade de la gestion. París: Seuil.

8. De la Garza, M.I., Medina, J.M., Cheín, N.F., Jiménez, K.P., Ayup, J. y Díaz, J.G. (2011). Los valores familiares y la empresa familiar en el Nordeste de México. Cuadernos De Administración, 24(42). https://www.redalyc.org/pdf/205/20520042015.pdf

9. Donnelley, R. (1964): “The Family Business", Harvard Business Review, Vol. 42, No. 2, pp. 93 105.

10. Esquivel Hernández, Javier; Martínez Prats, Germán y Silva Hernández, Francisca. Clima organizacional. Aspectos básicos de su origen y definición. Revista Ciencias de la Documentación, Vol: 6 núm 2 (2020): 66-77.

11. Fortino, S., Jeantet, A. y Tcholakova, A. (2015) “Emociones en el trabajo, trabajo de emociones", $L a$ 
nouvelle revue du travail, 6 . https://doi.org/10.4000/nrt.2071

12. Friedmann, Georges (1977). La crisis del progreso, Barcelona, Laia

13. Gallo, M. A. y Sveen, J. (1991). Internationalizing the family business: Facilitating and restraining factors. Family Business Review, 4(2), 181-190.

14. Garibaldi, A.V., Wetzel, U. Ferreira, V. (2009), Organizational culture and performance: a

15. Brazilian case, Management Research News, Vol. 32 Iss: 2 pp. $99-119$.

16. https://doi.org/10.1108/01409170910927578

17. Guerrero, P. y De Gaulejac, V. (2017). Clínicas del trabajo. Teorías e intervenciones. Santiago de Chile, Ediciones Universidad Alberto Hurtado. Recuperado de: http://ediciones.uahurtado.cl/wpcontent/uploads/2017/11/Clinicas-del-trabajo.pdf

18. Haider, S., Jabeen, S. y Ahmad, J. (2018). Moderated mediation between work life balance and employee job performance: The role of psychological wellbeing and satisfaction with coworkers. Revista de Psicología del Trabajo y de las Organizaciones, 34(1), 29-37. https:// dx.doi.org/10.5093/jwop2018a4

19. Maugeri, Salvatore. Vincent de Gaulejac, Travail, les raisons de la colère », La nouvelle revue du travail http://journals.openedition.org/nrt/439

20. Mayo, E. (1972). Problemas humanos de una civilización industrial, Buenos Aires: Nueva Visión.

21. Molina, P. A., Botero, S. y Montoya, J.N. (2016) Empresas de familia: conceptos y modelos para su análisis. Pensamiento y Gestión, núm. 41, 2016, pp. 116-149. ISSN 1657-6276

22. Navarro, J., Roe, R.A. y Artiles, M.I. (2015). Taking time seriously: changing practices and perspectives in Work/Organizational Psychology. Revista de Psicología del Trabajo y de las Organizaciones, 31(3), 135-145. https://dx.doi.org/10.1016/j.rpto.2015.07.002

23. Palafox, M.O., Ochoa, S. y Jacobo, C. A. (2019). La cultura organizacional como base para la permanencia en las organizaciones. Revista San Gregorio, [S.1.], n. 35, dic. 2019. ISSN 2528-7907.

24. http://dx.doi.org/10.36097/rsan.v1i35.1109

25. Palenzuela, P., Delgado, N. y Rodríguez, JA (2019). Explorando la relación entre el desempeño contextual y el agotamiento en los profesionales de la salud. Revista de Psicología del Trabajo y de las Organizaciones, 35, 115-121. https://doi.org/10.5093/jwop2019a13

26. Reyes, J. y Moros, H. (2019). La cultura organizacional: principales desafíos teóricos y metodológicos para su estudio. Revista Estudios del Desarrollo Social: Cuba y América Latina, 7(1), 201-217.

27. http://scielo.sld.cu/scielo.php?script=sci_arttext\&pid=S2308-01322019000100201\&lng=es\&tlng =es.

28. Rivera, D.A., Carrillo, S.M., Forgiony, J.O., Nuván, I.L. y Rozo,A.C. (2018) Cultura organizacional, retos y desafios para las organizaciones saludables. Vol. 39 (N22) Año 2018. Pág. 27.

29. http://www.revistaespacios.com/a18v39n22/18392227.html

30. Quiroz, J.O. (2010). Taylorismo, fordismo y administración científica en la industria automotriz. En Gestión y estrategia: A 100 Años de la administración científica. Repensando a Taylor. Año 19, número 38, (p.75-88). http://hdl.handle.net/11191/2960

31. Salgado, J.F., Blanco, S. y Moscoso, S. (2019). Bienestar subjetivo y desempeño laboral: Prueba de un efecto supresor. Revista de Psicología del Trabajo y de las Organizaciones, 35, 93-102. https:// doi.org/10.5093/jwop2019a9

32. Sampieri, R., Valencia, S. y Soto, R. (2014). Construcción de un instrumento para medir el clima organizacional en función del modelo de los valores en competencia. Contaduría y administración, 59(1), 229-257. https://dialnet.unirioja.es/servlet/articulo?codigo=5118462

33. Schein, E. H. (2004). Organizational culture and leadership (3ra. Ed.). San Francisco:

34. Jossey.Bass. ISBN 0-7879-6845-5

35. Urbiola, A.E. y Levin, S. (2018). Una propuesta para el estudio de la comunicación organizacional y la construcción de significados desde lo procesual y simbólico. Administración y Organizaciones, 21 (40), 51-72.

36. Zulmira, E., Mendes, A.M., Siqueira, M.V. y Garcia, J.N. (2009). Sedução e Servidão em um Caso de LER/DORT: diálogo entre a Psicodinâmica do Trabalho e a Sociologia Clínica. Psicologia Política, 9(18), 313-330.https://dialnet.unirioja.es/servlet/articulo? codigo=5061012 